\title{
Some Thoughts About Reporting the Electrocatalytic Performance of Nanomaterials
}

\author{
Danlei Li, Christopher Batchelor-McAuley, Richard G. Compton*
}

Physical and Theoretical Chemistry Laboratory, Department of Chemistry, University of Oxford, South Parks Road, Oxford OX1 3QZ, United Kingdom

*Corresponding author:

Email: Richard Compton Richard.compton@chem.ox.ac.uk

Phone: +44(0) 1865275957

Fax: $+44(0) 1865275410$

\begin{abstract}
In reading the ever expanding literature on electrocatalysts, we have become startled by the weakness of the electrochemistry often presented, which in some (many?) cases entirely negates the value of the work. In particular, we have been stimulated to consider the topic of this article by an Editorial (D. Voiry et al., 2018) in ACS Nano which recently provided 'guidance' on the 'best practices' for the measuring and reporting the activity of new electrocatalytic materials. From an electrochemical perspective, at least, contrasting views need to be presented since the suggestions provided are, in places, at odds with conventional wisdom or, more bluntly stated, simply wrong! In the following we do not seek to provide an alternative set of 'best practice guidelines' nor a 'set of materials characterisation requisites' - this is likely ultimately an appropriate activity for an IUPAC committee - but rather correct, amplify and develop the discussion provided by the editors of ACS Nano highlighting areas where we believe additional input is desirable and helpful. We focus on six topics that relate to recommendations made. In each section we start by making a brief statement that we believe is correct but different to that made by D. Voiry et al. This statement is then followed by a more in depth discussion and exploration of the issue at hand.
\end{abstract}

\section{Key words}

Electrocatalysis; electrochemical characterization; Tafel analysis; nanomaterials, characterization of 


\section{Standard, Formal and Equilibrium Potentials}

The standard redox potential is $\underline{\text { not }}$ under most conditions equivalent to the equilibrium potential. The standard potential is the equilibrium electrode potential defined at standard conditions (STP) with all the electroactive species at unit activity and gases at standard pressure (assuming ideal behaviour of the gas).

The following definitions are vital to aspects discussed later. To maintain a level of clarity we focus on the example of a simple one electron transfer process as given by:

$\mathrm{A}(\mathrm{sol})+\mathrm{e}^{-}=\mathrm{B}(\mathrm{sol})$

For this reaction the Nernst equation for the electrode potential $(\mathrm{E})$ is:

$E=E_{A / B}^{\ominus}-\frac{R T}{F} \ln \frac{a_{B}}{a_{A}}$

where $a_{\mathrm{i}}$ is the activity for the $i$ th species and $E_{A / B}^{\ominus}$ is the standard redox potential of the A/B redox couple. This latter value is directly related to the standard Gibbs energy of the reaction. If we express the solution phase activities of these electroactive solutes in terms of their activity coefficients on a concentration basis then using the expression $a_{i}=\gamma_{i} c_{i} / c^{\ominus}$, where $c^{\ominus}$ is the standard concentration $\left(1 \mathrm{~mol} \mathrm{dm}^{-3}\right)$ we can write: ${ }^{1}$

$E=E_{A / B}^{\ominus}-\frac{R T}{F} \ln \frac{\gamma_{B}}{\gamma_{A}}-\frac{R T}{F} \ln \frac{c_{B}}{c_{A}}$

where $\gamma_{i}$ is the activity coefficient, and the formal (aka conditional) potential $\left(E_{f, A / B}^{\ominus}\right)$ of the $\mathrm{A} / \mathrm{B}$ couple is then:

$E_{f, A / B}^{\ominus}=E_{A / B}^{\ominus}-\frac{R T}{F} \ln \frac{\gamma_{B}}{\gamma_{A}}$

First, the formal potential is not a fixed value solely dependent on the A/B couple but is specific to a given set of experimental conditions. If the activity coefficients of the electroactive species change, the formal potential is correspondingly altered. For this one-electron case only if $\frac{\gamma_{B}}{\gamma_{A}}=1$ does the standard potential equal the formal potential. Second, in contrast to the above case where both the reactant and product are solutes, if the reaction involves a gas then the standard and formal potentials often differ markedly. ${ }^{2}$ This arises due to the fact that most non-polar gases (such as hydrogen or oxygen) have a low solubility in aqueous solution. ${ }^{3}$ Hence, at one bar pressure the gas is commonly only present in solution at millimolar concentrations; in contrast, the formal potential is defined at (a possibly hypothetical) ${ }^{4}$ unit concentration. As such the solution phase dissolved gas concentration is often three orders of magnitude different between these two definitions. 
For a simple one-electron transfer process we can define the equilibrium potential as: ${ }^{5}$

$E_{e q, A / B}=E_{f, A / B}^{\ominus}-\frac{R T}{F} \ln \frac{c_{B}}{c_{A}}$

Even for this simple one-electron transfer reaction, Equation 5 is explicitly sensitive to the ratio of the oxidized (A) and reduced (B) species. If a solution contains one order of magnitude higher concentration of the product as compared to the reactant the equilibrium potential will be $\sim 59.1$ $\mathrm{mV}$ negative of the formal potential of the system. This may seem a moot point but the differences between these definitions become even more pronounced if an electrode reaction involves multiple steps. For example if the reaction involves the transfer of one proton per electron then the equilibrium potential varies with $59.1 \mathrm{mV} \mathrm{pH}^{-1}$. Electrocatalytic experiments performed at near neutral $\mathrm{pH}$ are often far from being under standard conditions!

\section{How Should We Quantify Electrode-kinetics?}

For a half-cell reaction the idea of the exchange current $\left(i_{0}\right)$ is only directly relevant in practical situations where both the electroactive product and reactant are present in the bulk solution phase and the reaction is to some extent reversible. Consequently, we cannot use io as parameter for generally defining electrocatalytic activity.

In electrochemistry there are two common formulations of the Butler-Volmer equation. First the more general form used by physical electrochemists which is close to that first derived by ErdeyGruz and Volmer: ${ }^{6}$

$i=-F A k_{A / B}^{0}\left(\mathrm{c}_{A, 0} \exp \left(\frac{-\alpha F}{R T}\left(E-E_{f, A / B}^{\ominus}\right)\right)-c_{B, 0} \exp \left(\frac{\beta F}{R T}\left(E-E_{f, A / B}^{\ominus}\right)\right)\right)$

$i$ is the current (A), $\mathrm{k}^{0}$ is the standard (strictly "formal" ${ }^{7}$ ) electrochemical rate constant ( $\mathrm{m} \mathrm{s}^{-1}$ ) and the $\mathrm{c}_{\mathrm{i}, 0}$ is the concentration of the $i$ th species at the electrode surface. The second form, originating from work by Laidler et al., ${ }^{8}$ is regularly employed in the materials and engineering literature and is given by the expression:

$i=-i_{0, A / B}\left(\exp \left(\frac{-\alpha F}{R T}\left(E-E_{e q, A / B}\right)\right)-\exp \left(\frac{\beta F}{R T}\left(E-E_{e q, A / B}\right)\right)\right)$

where $i_{0, A / B}$ is the exchange current (A) for the $\mathrm{A} / \mathrm{B}$ couple. Both expressions assume that $\alpha+$ $\beta=1$. The first major difference between these two equations ( 6 and 7$)$ is the potential against which they are referenced; in the first the potential is measured relative to the formal potential (Eq. 4) but the second uses the equilibrium potential (Eq. 5). Equation 7 can be derived from Equation 6 by rearranging the definition of the equilibrium potential (Eq. 5) to give a definition of the formal potential in terms of the equilibrium potential and by substituting this definition 
into Equation 6. Further for Equation 7 the surface concentration terms $\left(c_{i, 0}\right)$ are assumed equal to their value in bulk. At the equilibrium potential the anodic and cathodic currents are equal in size but of opposite direction summing to zero current. At this potential the current in either the anodic or cathodic direction is given by:

$i_{0, A / B}=F A k_{A / B}^{0} c_{A}^{1-\alpha} c_{B}^{\alpha}$

This so called "exchange current" has little physical significance; the rate of the reaction is controlled by both $k_{A / B}^{0}$ and $\alpha$ as expressed by Equation 6 .

Implicit in the use of Equation 7 is the assumption that both the reduced and oxidized species are present in the bulk solution phase; as is the case for classical work ${ }^{9}$ on the proton/hydrogen redox couple where both acid and dissolved hydrogen gas are present: $\mathrm{H}^{+}+\mathrm{e}^{-} \rightleftarrows \frac{1}{2} \mathrm{H}_{2}$. D. Voiry et al. emphasize the importance of estimating/measuring the exchange current $\left(i_{0}\right)$, as was undertaken classically, to quantify a the activity of catalysts. ${ }^{10}$ For the $\mathrm{H}^{+} / \mathrm{H}_{2}$ reaction we can usefully define and measure the associated exchange current $\left(i_{0}\right)$. But, we can see even for the one-electron example given above, if the concentration of product $\left(c_{B}\right)$ is zero then neither the equilibrium potential (Eq. 5) nor the exchange current (Eq. 8) are defined!

If only the reactant is present in bulk solution we face the question, how do we measure $\mathrm{i}_{0}$ ? This is a very commonly encountered situation, for instance when studying the hydrogen evolution reaction or the reduction of carbon dioxide where often neither hydrogen nor the products (formate, oxalate, carbon monoxide etc.) of the carbon dioxide reduction process are initially present in the bulk solution phase. Hence, any attempts to provide general guidelines for quantifying the properties of electrocatalytic materials, which are predicated on the measurement of a quantity (io) that is only relevant to a special situation, is not helpful. Rather for individual half reactions, $A+e^{-} \rightarrow B$ or $A-e^{-} \rightarrow B$, the measurement of $\mathrm{k}^{0}$ and $\alpha$ (or equally [1- $\alpha$ ]) is preferred.

We note that at any given potential the Principle of Microscopic Reversibility ${ }^{11}$ requires that the anodic and cathodic reactions have the same transition state; consequently, for a one-electron transfer process $\alpha+\beta=1$ when measured at the same potential. However, for couples with significant irreversibility the values of $\alpha$ and $\beta$ are commonly evaluated at different potentials for the cathodic and anodic processes respectively. Since the Principle of Microscopic Reversibility only rigorously holds at the same potential, when $\alpha$ and $\beta$ are measured at different potentials they need not add to unity. At other potentials the relative magnitudes of $\alpha$ and $\beta$ may vary as the character of the transition state changes, for example as solvation/adsorption or the double layer structure alters with the applied potential. Thus generally for experimentally measured transfer coefficients $\alpha+\beta \neq 1$. Hence, linear extrapolation of the cathodic and anodic branches of a voltammograms to give $\mathrm{E}_{\mathrm{eq}}$ and $\mathrm{i}_{0}$ will be in error. 
Finally, for multi-step reactions the mechanism and hence the associated reaction product is often potential dependent. Consider two electrode reactions that occur in parallel, a hypothetical example might be the reduction of $\mathrm{CO}_{2}$ to either $\mathrm{CO}$ or formate. If the formation of say, for example, $\mathrm{CO}$ on some new catalysts has a (comparatively) low standard electrochemical rate constant but a high transfer coefficient and the other reaction, in this case leading to the formation of formate, has a high standard electrochemical rate constant but a low transfer coefficient then the electrochemical reaction product will be potential dependent. At low potentials the reaction with the higher standard electrochemical rate constant will dominate and in this example we will yield formate as a product. Conversely at high overpotentials the relative rates of these two processes will have switched and the dominant reaction product will be the formation of $\mathrm{CO}$. For multi-step processes the nature of the electrode reaction mechanism will often change as a function of the applied potential!

\section{What is an Overpotential?}

In the case where only the electroactive reagent is present in solution and under a steady-state mass-transport regime we can use the shift in the voltammetric half-wave potential from that expected for a reversible process as a measure of the applied overpotential. However, precise calculation of this expected reversible half-wave potential for a given set of experimental conditions is not necessarily facile; this is especially true for multistep reactions. ${ }^{2}$

The problems outlined in the previous section relating to the exchange current also underlie an issue in the IUPAC definition of overpotential. IUPAC rigidly define the overpotential in relation to the equilibrium potential $\left(\eta_{e}=E-E_{e q}\right)^{5}$ but from Equation 5 if either $\mathrm{c}_{\mathrm{A}}$ or $\mathrm{c}_{\mathrm{B}}$ is zero then the overpotential by this definition cannot be defined! This does not however imply that the thermodynamics of such a system are also ill-defined, we just need a different definition. Generally, if an electrochemical process is described as 'reversible' under a given mass-transport regime this implies that the electrode surface concentrations of the reduced and oxidized species are well described via the Nernst equation (i.e. they are locally at equilibrium).

In the literature it is common in cases where only the reactant is present in the bulk solution to define the overpotential relative to that of the formal potential $\left(\eta_{f}=E-E_{f}^{\ominus}\right)$ or even in some cases the standard potential $\left(\eta_{s}=E-E^{\ominus}\right)$. For a simple one-electron transfer process this definition of overpotential is clear and rational with Equation 6 as the inspiration. For example if we consider the reversible one-electron oxidation of ferrocene methanol: $\mathrm{Fc}-\mathrm{e}^{-} \rightleftarrows \mathrm{Fc}^{+}$. Under steady-state mass-transport conditions then the voltammetric half-wave ${ }^{12}$ potential is approximately equal to the formal potential. In cases where the diffusion coefficients of the reduced and oxidized species are equal then the reversible half-wave potential $\left(E_{1 / 2}\right)$ is exactly equal to the formal potential of the system. For the hydrogen evolution reaction $\left(\mathrm{H}^{+}+\mathrm{e}^{-} \rightleftarrows\right.$ 
$\frac{1}{2} \mathrm{H}_{2}$ ) due to the process involving the formation and breaking of chemical bonds the situation is slightly different. Here we briefly consider the case of the hydrogen evolution reaction from an aqueous strong acid solution in the absence of dissolved hydrogen in the bulk phase. The Nernst equation describing the electrode potential can be expressed as:

$E=E_{f, H^{+} / H_{2}}^{\ominus}+\frac{R T}{F} \ln \frac{c_{H^{+}, 0}}{c_{H_{2,0}} 0.5 \vartheta^{0.5}}$

where the square-root associated with the concentration of hydrogen reflects the stoichiometry of the reaction. Under steady-state conditions, for example as obtained using a rotating disc electrode, at the voltammetric half-wave potential i.e. where $50 \%$ of the available protons are converted to hydrogen - and again assuming equal diffusion coefficients for the electroactive species - then the surface concentrations of the reduced (hydrogen) and oxidized (protons) species will be equal to:

$c_{H^{+}, \text {bulk }} \approx 2 c_{H^{+}, 0} \approx 4 c_{H_{2}, 0} \quad$ at $E=E_{1 / 2}$

where the subscript zero refers to the surface concentrations of the species. Equation 10 expresses that at the voltammetric half-wave potential the surface concentration of the protons $\left(c_{H^{+}, 0}\right)$ will be half of that in the bulk media $\left(c_{H^{+}, \text {bulk }}\right)$. Moreover, on the basis of the reactions stoichiometry the reduction of half of the protons to hydrogen at the electrode surface implies that the surface concentration of hydrogen $\left(c_{H_{2}, 0}\right)$ is equal to a quarter of the bulk proton concentration. Substitution of equality 10 into Equation 9 yields an expression for the voltammetric half wave potential $\left(E_{1 / 2}\right)$ as equal to:

$E_{1 / 2} \approx E_{f, H^{+} / H_{2}}^{\ominus}+\frac{R T}{F} \ln \frac{c_{H^{+}, b u l k}^{0.5}}{c^{0.5}}$

Equation 11 is only exactly correct in the case that the diffusion coefficients of the reduced and oxidized species are equal. ${ }^{13}$ However the salient point is that, as a result of conservation of mass at the electrode surface and due to the reactions stoichiometry, the position of the reversible voltammetric wave is, as measured by the voltammetric half-wave potential, related to the formal potential for the reaction but varies as a function of bulk acid concentration! ${ }^{14}$

\section{What is an Onset Potential?}

The onset potential is neither a thermodynamically nor kinetically well-defined parameter; consequently, it is unhelpful when comparing activity of electrocatalysts between laboratories and hence across different experimental setups. A likely more appropriate method is, as is done with ORR catalysts, to report the Faradaic current density at a given and agreed potential. ${ }^{15}$

Although the "onset potential" is a widely reported parameter and at first sight appears to have an obvious definition ${ }^{16}$ as something akin to the "lowest overpotential at which a reaction product 
is formed at a given electrode under defined conditions' or 'the lowest overpotential at which the Faradaic current is observed to be over and above the measured background' these statements belie the true complexity of the issue as the following explains. First, consider an irreversible one-electron reduction process for which:

$i=F A k_{A / B}^{0} \exp \left(\frac{-\alpha F\left(E-E_{f, A / B}^{\theta}\right)}{R T}\right) c_{A, 0}$

The cathodic current is predicted to asymptotically approach zero as $E \rightarrow+\infty$ and to increase exponentially as $E \rightarrow-\infty$. Defining where such an exponential rise 'starts' necessarily requires some additional arbitrary definitions. Second, the general interpretation of the onset potential is the potential at which a reductive or oxidative faradaic reaction becomes measureable. Immediately it becomes clear that such a parameter is essentially a measure of the signal-to-noise or signal-to-background ratio of the system and hence does not solely reflect the electrocatalytic properties of the material under study but is also influenced by the measurement conditions. To exemplify this point Figure 1 shows a simulated one-electron irreversible reduction at a rotating disc electrode. The employed simulation numerically calculates the voltammetric profile using a fully implicit finite difference method and makes use of the Hale transform. ${ }^{17,}{ }^{18}$ The theoretically predicted Faradaic current is plotted in black. Also plotted are some realistic values for the capacitative current of the electrode. In particular we have approximated the electrodes capacitance as constant with respect to the applied electrode potential and supposed that the specific capacitance has value that is representative for metals in aqueous solutions $\left(20 \mu \mathrm{F} \mathrm{cm}^{-2}\right.$ )$^{19}$

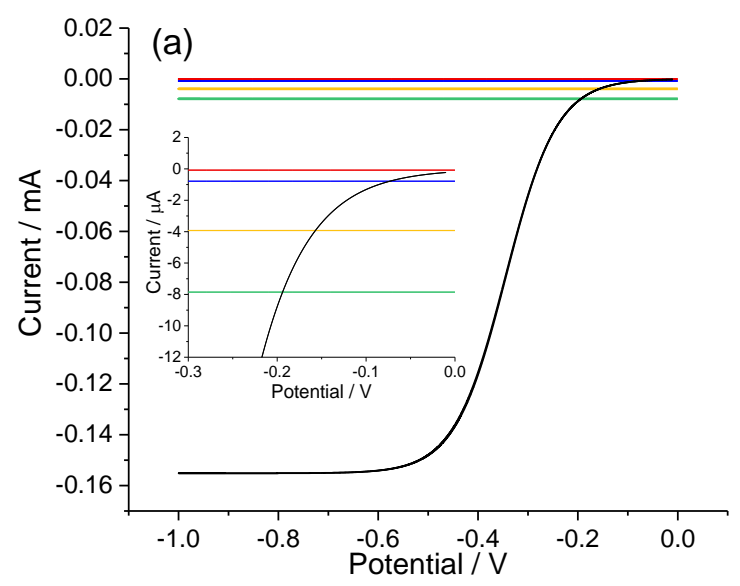

(b)

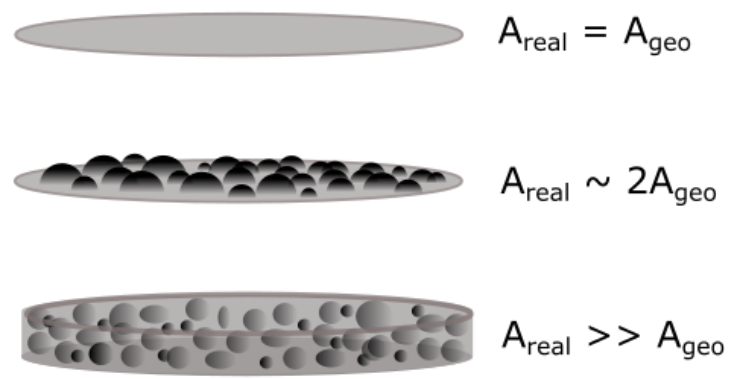

Figure 1: (a) Comparison of the simulated Faradaic current (black) and capacitative current at a rotating disc electrode $\left(5 \mathrm{~mm}\right.$ in diameter) with variable roughness factors $\left(R_{f}\right), R_{f}=1$ (red), $R_{f}=10$ (blue), $R_{f}=50$ (yellow) and $R_{f}=100$ (green). Inlay is the zoomed-in version with a potential range from $-0.3 \mathrm{~V}$ to $0 \mathrm{~V}$. Parameters in the simulation: scan rate $\mathrm{v}=0.02$ $\mathrm{V} \mathrm{s}^{-1}$, rotation rate $\omega=1600 \mathrm{rpm}$, concentration $\mathrm{c}=1 \mathrm{mM}$, diffusion coefficient $\mathrm{D}=1 \times 10^{-9} \mathrm{~m}^{2} \mathrm{~s}^{-1}$, formal electron transfer rate constant $\mathrm{k}^{0}=1 \times 10^{-7} \mathrm{~m} \mathrm{~s}^{-1}$, formal potential $\mathrm{E}_{\mathrm{f}}=0 \mathrm{~V}$, viscosity $=8.9 \times 10^{-7} \mathrm{~m}^{2} \mathrm{~s}^{-1}$, transfer coefficient $\alpha=\beta=$ 0.5. The capacitative current $I_{c a p}=C_{d l}\left(20 \mu F \mathrm{~cm}^{-2}\right) \times R_{f} \times A_{g e o} \times v$. (b) Schematic of electrode surface with different roughness factors. The roughness factor $\mathbf{R}_{\mathbf{f}}=$ the real electrode area $\left(\mathbf{A}_{\text {real }}\right) /$ the geometric area $\left(\mathbf{A}_{\text {geo }}\right)$. 
To calculate the total electrode area we have multiplied the simulated geometric electrode ( $\mathrm{A}_{\text {geo }} /$ $\mathrm{m}^{2}$ ) area by a roughness factor, $\mathrm{R}_{\mathrm{f}}$, so that $\mathrm{A}_{\text {real }}=\mathrm{A}_{\text {geo }} \mathrm{x} \mathrm{R}_{\mathrm{f}}$. As discussed later, for heterogeneous electrode surfaces another important quantity is the roughness factor which accounts only for the real area of the catalyst $\left(\mathrm{R}_{\mathrm{f} \text {,catalyst }}\right)$. As outlined schematically in Figure $\left.1 \mathrm{~b}\right)$ in the present example a perfectly atomically flat electrode would have a roughness factor of unity; a very well prepared polycrystalline electrode may be expected to have a roughness factor of $\sim 1.5^{15}$ and a thin-film modified RDE will have a widely variable roughness factor in the range of 10-1000. Thin-film modified electrodes find routine use in electrocatalyst experiments,${ }^{15}$ where a catalyst is mixed with a non-catalytic conductive support such as a form of nano-carbon and added as a thin-layer across the surface of the electrode. The capacitative charging of the carbon support and the catalyst will both contribute to the total background charging currents. As can be seen in the guidelines provided by Kocha et al. ${ }^{15}$ in their experiments for a thin-film modified RDE of diameter $5 \mathrm{~mm}$ the total background currents are of the order of $15 \mu \mathrm{A}$, this corresponds to an effective roughness factor of approximately 190 .

From Figure 1 a) we can see that if we define the onset potential in this model case as when the Faradaic current is expected to be equal in magnitude to the capacitative contribution then the potential at which this cross over occurs is extremely sensitive to the capacitance of the electrode. Even in this idealized case the value of the onset potential varies by more than $200 \mathrm{mV}$ for these realistic electrode capacitances. We further comment that the definition of the capacitance as being a constant, as is implied in the original editorial text, and as is used above is an oversimplification. ${ }^{20}$

\section{What is the Appropriate Tafel Region of the Current-Potential Plot of a Half-Cell Reaction in which to Analyse a 'Tafel-Slope'?}

It is preferable to define a current range relative to the limiting steady state value as opposed to defining a suitable potential range for meaningful Tafel analysis giving either a transfer coefficient or, equivalently, a 'Tafel slope'. The analysis provided here indicates that provided a suitable background subtraction to remove the capacitative current contribution is first performed then the current in the range between 10-80\% of the limiting current is suitable for kinetic analysis once a mass-transport correction has been made. If the latter correction is neglected only currents below $\sim 20 \%$ of the steady-state current are suitable for use.

In a Tafel plot, the $\log$ of the current, $\log _{10}|\mathrm{i}|$, is plotted against the applied potential, E. If we assume the reaction is fully irreversible and well described by the Tafel equation (Eq. 12) then we can see for a one-electron transfer process that such a semi-log plot will have a slope that is equal to $-\alpha F /(R T \times \ln 10)$ and an intercept (at zero overpotential, $\left.\eta_{f}\right)$ equal to $\log _{10}\left(F A k^{0} c_{A}\right)$. Originally electrocatalysis experiments were performed using a form of current-interrupt technique known as the commutator method. ${ }^{21,22}$ Here a desired current density was driven to 
occur on a working electrode, the cell was subsequently disconnected and the rapidly changing cell potential measured against a reference cell. Measurement of the potential of the disconnected cell as a function of the disconnection time allowed the original cell potential to be inferred by extrapolation. Hence in reporting data in terms of current/potential graphs on the y-axis the potential (the measured variable) was plotted against the current (the controlled variable). ${ }^{22}$ Modern experiments tend to be performed potentiometrically and hence one might expect the axes of the Tafel plot to be swapped, this is virtually never the case; old habits die hard. For more information on alternate methods of presenting Tafel plots and analysis see ref $\left[{ }^{23}\right]$.

A linear Tafel plot of $\log _{10}|\mathrm{i}|$ vs $\mathrm{E}$ requires that, on the basis of Equation 6, the process is fully irreversible and that the surface concentrations of the electroactive species remain constant throughout the potential range of analysis. In practice the replenishment of the active species via diffusion is slow and distortions arise from mass-transport limitations. Consequently, it is necessary to 'correct' for these changes in the redox active species at the electrode surface. The rotating disc electrode is to a reasonable approximation ${ }^{24,25}$ uniformly accessible. Consequently a plot of $\log _{10}\left(1 / \mathrm{I}-1 / \mathrm{I}_{\text {lim }}\right)$ against the applied potential allows for these changes in the surface concentration of the redox active species during the course of the scan to be suitably accounted for. The need to make a mass-transport correction has long been advocated. ${ }^{26,27}$

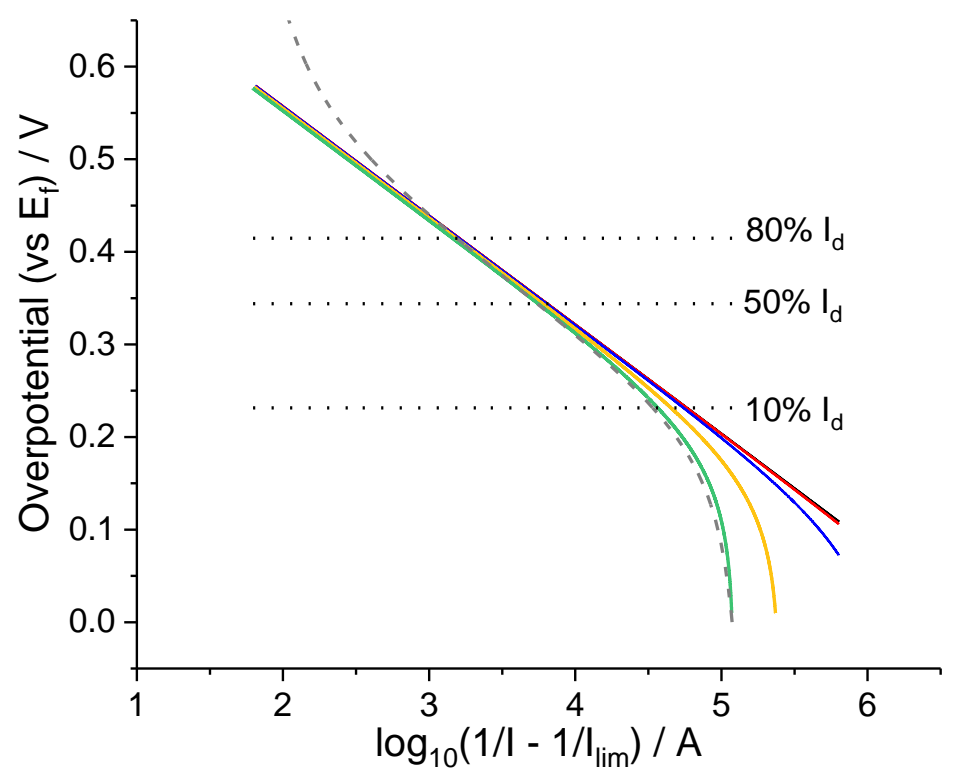

Figure 2 Simulated mass-transport corrected Tafel plots for a rotating disc electrode. Solid lines represent the electrode with constant capacitances with variable roughness factors $R_{f}: R_{f}=0$ (black), $R_{f}=1$ (red), $R_{f}=10$ (blue), $R_{f}=50$ (yellow) and $R_{f}=100$ (green). The capacitative current $I_{c a p}=C_{d l}\left(20 \mu \mathrm{F} \mathrm{cm}^{-2}\right) \times R_{f} \times A_{g e o} \times v$. Dashed line stands for the electrode with a variable capacitative current (grey dashed) where the variable capacitative current was calculated from $I_{c a p}=C_{d l}$ $\left(20 \mu \mathrm{F} \mathrm{cm}^{-2}\right) \times \mathbf{R}_{\mathbf{f}} \times A_{\text {geo }} \times \mathbf{v} \times \mathbf{f}(\mathrm{E})$, where $\mathbf{E}$ is the electrode potential. Simulation parameters: scan rate $\mathbf{v}=\mathbf{0 . 0 2} \mathrm{V} \mathrm{s} \mathbf{s}^{-1}$, rotation rate $\omega=1600 \mathrm{rpm}$, concentration $\mathrm{c}=1 \mathrm{mM}$, diffusion coefficient $\mathrm{D}=1 \times 10^{-9} \mathrm{~m}^{2} \mathrm{~s}^{-1}$, formal electron transfer rate constant $\mathrm{k}^{0}=1 \times 10^{-7} \mathrm{~m} \mathrm{~s}^{-1}$, formal potential $E_{f}=0 \mathrm{~V}$, viscosity $=8.9 \times 10^{-7} \mathrm{~m}^{2} \mathrm{~s}^{-1}$, transfer coefficient $\alpha=\beta=0.5$. I tot $=I_{\text {farad }}$ $+\mathbf{I}_{\text {cap. }}$. 
Figure 2 plots an example mass-transport corrected Tafel plot for a simulated RDE experiment. In this simulation we have assumed that the total current can be expressed simply as the sum of both the Faradaic and non-Faradaic contributions $\left(i_{\text {tot }}=i_{\text {farad }}+i_{\text {cap }}\right)$. For the solid lines in Figure 2 the contribution of the capacitance is assumed to be a constant ( $i_{\text {cap }}=C_{d l} \times R_{f} \times A_{\text {geo }} \times v$ ), alternatively the dotted line assumes that the capacitance of the electrode varies linearly as a function of the applied potential, $\mathrm{i}_{\text {cap }}=\mathrm{C}_{\mathrm{dl}} \times \mathrm{R}_{\mathrm{f}} \times \mathrm{A}_{\text {geo }} \times v \times \mathrm{f}(\mathrm{E})$, where the function $\mathrm{f}(\mathrm{E})$ is a dimensionless scalar that varies linearly between 1 and 2 across the simulated voltammetric potential range. In the absence of a background correction to removes the capacitative current Figure 2 shows how the mass-transport corrected Tafel plot is distorted by the presence of a capacitative "background" current. Capacitative currents are symptomatic of the used voltammetric technique. This result first highlights the importance of background correction to remove the non-Faradaic contribution from the voltammetric data. It also gives a clear indication as to how sensitive the data will be to the quality of this correction. It is on this basis that Mayrhofer et al. previously proposed that the kinetically useful part of an RDE voltammogram is the mass-transport corrected current between $10-80 \%$ of the steady-state value ${ }^{27}$ Kocha et al. ${ }^{28}$ were more cautious and on the basis of work by Vidal-Iglesias et al. ${ }^{29}$, advised that only the 10$50 \%$ current regime is useable.

\section{Units and Electrochemical Surface Areas}

The size of the electrochemically available surface area of the catalyst is an important factor in determining meaningful information regarding its catalytic abilities if electron transfer (not mass-transport) is rate determining. Two chemically relevant quantities are the roughness factor (a dimensionless measure of the surface area of the catalyst per geometric area of the electrode) and the electrochemical surface area (the surface area of the catalyst per gram of catalyst). Measurement of the real surface area of the catalyst is often experimentally challenging and is in some cases sensitive to how the measurement is made.

D. Voiry et $a l^{30}$ present both the electrochemical surface area (ECSA) and the turnover frequency (TOF) as dimensionless parameters! In the guidelines the ECSA is given as the dimensionless ratio of the specific double layer capacitance $\left(\mathrm{C}_{\mathrm{dl}}\right)$ relative to a given reference surface specific capacitance, this definition seems to follow a previous article in ACS Catalysis. ${ }^{31}$ Similarly the TOF is given as " $n_{\text {product }} / n_{\text {site }}$ " $;{ }^{30}$ how these two terms ( $n_{\text {product }}$ and $n_{\text {site }}$ ) should be defined and measured is left to the reader. We note as follows, first, D. Voiry et al. have interpreted the ECSA as a roughness factor with a subsequent confusing conflation of terminology in the text. Second, we comment that it is essential for a surface area to contain the unit of length squared; similarly a quantity labelled as a "frequency" has units of reciprocal time. As a consequence of these non-conventional definitions some of the derived expressions provided D. Voiry et al. may also be usefully reconsidered. 
In the literature the definition of the ECSA may vary depending on the context in which it is being used; however, following the ORR field the ECSA is probably most usefully defined as the area of the catalyst per gram of material $\left(\mathrm{m}^{2} \mathrm{~g}_{\text {catalyst }}^{-1}\right){ }^{27}$ This value in combination with the specific activity of the catalyst (A $\mathrm{m}_{\text {catalyst }}^{-2}{ }^{32}$ the catalytic Faradaic current per catalyst area), yields the mass activity for the material ( $\mathrm{A} \mathrm{g}_{\text {catalyst }}^{-1}$ ). These latter two values vary as a function of potential. Consequently to get a relative measure of activity these values are often reported at a given potential. It is the value for the mass activity that most directly translates to the cost of a platinum based fuel cell device. ${ }^{33}$ But in terms of physico-chemical insight the important factor is arguably the specific activity of the catalyst. This value of the catalysts specific activity gives a measure of the rate of reaction per unit area of the catalyst, accurate determination of the magnitude of this value is not necessarily as facile as it may seem; this is especially true if the catalytic surface is not uniformly accessible as is the case for porous electrode surfaces. Related to these values is the (dimensionless) catalyst roughness factor, this value is the surface area of the catalyst per geometric area of the electrode. In some contexts the roughness factor may also, vide supra, refer to the total surface area of the electrode relative to its geometric area.

The measurement of the surface area of the catalysts is not necessarily straightforward. Note that "best practice" documents in the field of ORR tend to only very briefly ${ }^{15}$, if at all ${ }^{27}$, mention that such electrode capacitance measurements (or the measurement of other surface processes such as the under-potential deposition of hydrogen) need to be made using an analog ${ }^{34}$ potentiostat. In the case of measuring platinum electrochemical surface areas, major errors can be made if staircase voltammetry is used and, of course, almost all modern commercial potentiostats provide staircase voltammetry as the default technique, so this can lead to the underestimation of the platinum surface area and will hence cause an overestimation regarding the material's specific activity. As an aside similar issues arise with protein film voltammetry. ${ }^{35}$ This issue with staircase voltammetry is often covered in potentiostat manuals $;{ }^{36}$ but who reads a manual $?^{37}$

Some of the above is opinion and no doubt others in the field will dispute aspects of it. Neither is the above exhaustively critical of the editorial written by D. Voiry et al. ${ }^{30}$ Some comments will be obvious to many in the electrochemical field but we hope that we have identified some of the important points for the non-specialist.

\section{Conflict of interest}

The authors declare no competing financial interest.

\section{Acknowledgement}

D. Li thanks for the China Scholarship Council and the University of Oxford for supporting her DPhil research. 


\section{References}

1. In equation 3 the standard concentration terms have cancelled out, in cases where the stoichiometry of the reaction is not unity this is not the case.

2. Jiao, X.; Batchelor-McAuley, C.; Kätelhön, E.; Ellison, J.; Tschulik, K.; Compton, R. G., The subtleties of the reversible hydrogen evolution reaction arising from the nonunity stoichiometry. The Journal of Physical Chemistry C 2015, 119 (17), 9402-9410.

3. Wilhelm, E.; Battino, R.; Wilcock, R. J., Low-pressure solubility of gases in liquid water. Chemical reviews 1977, 77 (2), 219-262.

4. Note the formal potential is still relevant for understanding the thermodynamics of electrochemical processes involving dissolved gases.

5. Cohen, E. R.; Mills, I.; Royal Society of, C.; Cvitas, T.; International Union of, P.; Applied Chemistry, P.; Biophysical Chemistry, D.; Frey, J. G.; Quack, M.; Holström, B.; Kuchitsu, K.; Marquardt, R., Quantities, Units and Symbols in Physical Chemistry. Royal Society of Chemistry: 2007.

6. Erdey-Grúz, T.; Volmer, M., Zur theorie der wasserstoff überspannung. Zeitschrift für physikalische Chemie 1930, 150 (1), 203-213.

7. In strict accordance with IUPAC $\mathrm{k} 0$ as defined here is the formal electrochemical rate constant as the used reference potential is the formal not the standard potential see ref 6 .

8. Eyring, H.; Glasstone, S.; Laidler, K. J., Application of the theory of absolute reaction rates to overvoltage. The Journal of Chemical Physics 1939, 7 (11), 1053-1065.

9. Pentland, N.; Bockris, J. M.; Sheldon, E., Hydrogen evolution reaction on copper, gold, molybdenum, palladium, rhodium, and iron mechanism and measurement technique under high purity conditions. Journal of The Electrochemical Society 1957, 104 (3), 182-194.

10. Trasatti, S., Work function, electronegativity, and electrochemical behaviour of metals: III. Electrolytic hydrogen evolution in acid solutions. Journal of Electroanalytical Chemistry and Interfacial Electrochemistry 1972, 39 (1), 163-184.

11. Thomson, W., IX.-On the Dynamical Theory of Heat. Part V. Thermo-electric Currents. Transactions of the Royal Society of Edinburgh 1853-1857, 21 (Part 1), 123-171.

12. Meites, L.; Zuman, P.; Nurnberg, H. W., Recommended terms, symbols, and definitions for electroanalytical chemistry (IUPAC Recommendations 1985). In Pure and Applied Chemistry, 1985; Vol. 57, p 1491.

13. For the proton/hydrogen redox couple in aqueous solution the diffusion coefficients of the reduced and oxidised species differ by approximately a factor of two. This difference in the diffusion cofficients will, for a rotating disc electrode experiment, lead to the half-wave potential being shifted by approximately $-5 \mathrm{mV}$ from that predicted on the basis of Eq. (11). For more information on this additonal mass-transport correction to the predicted half-wave potential, the reader is directed towards ref 3 . In the SI of this reference the additionally required correction for the half-wave potential is derived (Eq. 12 of SI in ref.3), simply requiring the definition for the hydrodynamic layer thickness to be substituted into this expression.

14. It is important to recognise that this sensitivity to the bulk acid concentration is not the same as that of the so-called 'Reversible Hydrogen Electrode'. To this end we highlight the square-root present in the natural log term in Equation 11.

15. Kocha, S. S.; Shinozaki, K.; Zack, J. W.; Myers, D. J.; Kariuki, N. N.; Nowicki, T.; Stamenkovic, V.; Kang, Y.; Li, D.; Papageorgopoulos, D., Best Practices and Testing Protocols for Benchmarking ORR Activities of Fuel Cell Electrocatalysts Using Rotating Disk Electrode. Electrocatalysis 2017, 8 (4), 366-374. 
16. Maljusch, A.; Ventosa, E.; Rincón, R. A.; Bandarenka, A. S.; Schuhmann, W., Revealing onset potentials using electrochemical microscopy to assess the catalytic activity of gas-evolving electrodes. Electrochemistry Communications 2014, 38, 142-145.

17. Compton, R. G.; Laborda, E.; Ward, K. R., Understanding Voltammetry: Simulation Of Electrode Processes. World Scientific Publishing Company: 2013.

18. Hale, J. M., Transients in convective systems: I. Theory of galvanostatic, and galvanostatic with current reversal transients, at a rotating disc electrode. Journal of Electroanalytical Chemistry (1959) 1963, 6 (3), 187-197.

19. Damaskin, B. B.; Frumkin, A. N., Potentials of zero charge, interaction of metals with water and adsorption of organic substances-III. The role of the water dipoles in the structure of the dense part of the electric double layer. Electrochimica Acta 1974, 19 (4), 173-176.

20. Gerischer, H.; McIntyre, R.; Scherson, D.; Storck, W., Density of the electronic states of graphite: derivation from differential capacitance measurements. Journal of Physical Chemistry 1987, 91 (7), 1930-1935.

21. Newbery, E., Overvoltage. Journal of the Chemical Society, Transactions 1914, 105 (0), 2419-2435.

22. Bowden, F. P.; Rideal, a. E. K., The electrolytic behaviour of thin films. Part I.Hydrogen. Proceedings of the Royal Society 1928, 120 (784), 59.

23. Li, D.; Lin, C.; Batchelor-McAuley, C.; Chen, L.; Compton, R. G., Tafel analysis in practice. Journal of Electroanalytical Chemistry 2018, 826, 117-124.

24. Newman, J., Current distribution on a rotating disk below the limiting current. Journal of the Electrochemical Society 1966, 113 (12), 1235-1241.

25. Smyrl, W. H.; Newman, J., Limiting current on a rotating disk with radial diffusion. Journal of The Electrochemical Society 1971, 118 (7), 1079-1081.

26. $\quad$ Albery, W. J., Electrode kinetics. Clarendon Press: 1975.

27. Mayrhofer, K. J. J.; Strmcnik, D.; Blizanac, B. B.; Stamenkovic, V.; Arenz, M.;

Markovic, N. M., Measurement of oxygen reduction activities via the rotating disc electrode method: From Pt model surfaces to carbon-supported high surface area catalysts. Electrochimica Acta 2008, 53 (7), 3181-3188.

28. Shinozaki, K.; Zack, J. W.; Richards, R. M.; Pivovar, B. S.; Kocha, S. S., Oxygen reduction reaction measurements on platinum electrocatalysts utilizing rotating disk electrode technique: I. Impact of impurities, measurement protocols and applied corrections. Journal of the Electrochemical Society 2015, 162 (10), F1144-F1158.

29. Vidal-Iglesias, F. J.; Solla-Gullón, J.; Montiel, V.; Aldaz, A., Errors in the use of the Koutecky-Levich plots. Electrochemistry Communications 2012, 15 (1), 42-45.

30. Voiry, D.; Chhowalla, M.; Gogotsi, Y.; Kotov, N. A.; Li, Y.; Penner, R. M.; Schaak, R. E.; Weiss, P. S., Best Practices for Reporting Electrocatalytic Performance of Nanomaterials. ACS Nano 2018, 12 (10), 9635-9638.

31. Clark, E. L.; Resasco, J.; Landers, A.; Lin, J.; Chung, L.-T.; Walton, A.; Hahn, C.; Jaramillo, T. F.; Bell, A. T., Standards and protocols for data acquisition and reporting for studies of the electrochemical reduction of carbon dioxide. ACS Catalysis 2018, 8 (7), 65606570 .

32. Here $\mathrm{A}$ is the unit Ampere and not the symbol representing the electrode area which has units of $\mathrm{m} 2$. 
33. Kongkanand, A.; Mathias, M. F., The priority and challenge of high-power performance of low-platinum proton-exchange membrane fuel cells. The journal of physical chemistry letters 2016, 7 (7), 1127-1137.

34. Batchelor-McAuley, C.; Yang, M.; Hall, E. M.; Compton, R. G., Correction factors for the analysis of voltammetric peak currents measured using staircase voltammetry. Journal of Electroanalytical Chemistry 2015, 758, 1-6.

35. Heering, H. A.; Mondal, M. S.; Armstrong, F. A., Using the pulsed nature of staircase cyclic voltammetry to determine interfacial electron-transfer rates of adsorbed species. Analytical chemistry 1999, 71 (1), 174-182.

36. Autolab, M.-. Application Note: Comparison between Staircase Cyclic Voltammetry and Cyclic Voltammetry Linear Scan pp https://www.metrohm.com/en-gb/applications/AN-EC-007.

37. Alethea L. Blackler, R. G., Vesna Popovic and M. Helen Thompson, IG Nobel-

Literature Prize: for documenting that most people who use complicated products do not read the instruction manual. 2018. 